\title{
Industrial Fieldbus Improvements in Power Distribution and Conducted Noise Immunity With No Extra Costs
}

\author{
Alberto Menéndez, Antonio Barbancho, Enrique Personal, Student Member, IEEE, and Diego \\ Fco. Larios
}

\begin{abstract}
Industrial distributed control continues the move toward networks at all levels. At lower levels, control networks provide flexibility, reliability, and low cost, although perhaps the simplest but most important advantage is the reduced volume of wiring. Powered fieldbuses offer particular notable benefits in system wiring simplification. Nevertheless, very few papers are dealing with the potentials and limitations in power distribution through the bus cable. Only a few of the existent fieldbus standards consider this possibility but often simply as an option without enough technical specifications. In fact, nobody talks about it, but power distribution through the bus and conducted noise disturbances are strongly related. This paper points out and analyzes these limitations and proposes a new low-cost fieldbus physical layer that enlarges power distribution capability of the bus and improves system robustness. We show an industrial application on water desalination plants and the very good results obtained owing to the fieldbus. Finally, we present electromagnetic compatibility test results that verify improvements against electrical fast transients on the sensor/actuator connection side as disturbances usually encountered in harsh-environment industrial applications.
\end{abstract}

Index Terms-Bus conducted electric noise, common-mode disturbances at distributed $\mathrm{I} / \mathrm{O}$, control networks, distributed power supply, electrical fast transient disturbances, fieldbus physical layer, industrial automation, machine level control.

\section{INTRODUCTION}

$\mathbf{I}$ NDUSTRIAL automation has been applying networking technologies for many years now, but trends in manufacturing continue to move toward networks at all levels. Connecting the control system components via networks can effectively reduce the complexity of the system and enhance interconnectivity and remote control facilities. At lower levels in the factory infrastructure, current control and data acquisition systems for industrial process or for complex machinery have increasingly come to rely on fieldbus system architecture. The word fieldbus has been widely used for designing a control network connecting field devices such as sensors, actuators, regulators, power line carrier (PLC), etc., and human-machine interfaces. Fieldbus systems replace traditional point-to-point wired systems providing reduced volume of wiring and fewer connecting points, resulting in a reliability increase, improved capability of troubleshooting and maintenance, and enhanced interchangeability and interoperability of devices.

Nevertheless, fieldbus technology in industrial automation is relatively complex because of the number of possible solutions and because of the variety of applications: process control of continuous processes, discrete manufacturing plants, building automation, remote monitoring and control of utilities networks, transportation systems, etc. Companies, end users, and control manufacturers have realized the strategic importance of the fieldbus in the industrial automation so that, for about 20 years now, they began a standardization process [1] leading to a current state with many fieldbus standards having different sectorial and national supports but all of them with a solid market position: PROFIBUS, Foundation FIELBUS, WorldFIP, AS-i, CAN, DeviceNET, LON, INTERBUS, MODBUS, etc. They correspond to different approaches and solutions [2], dealing with different types and amounts of data to be transmitted, reflecting the very large spectrum of techniques and applications as well as commercial interest's rivalry.

Communication by serial data transmission on fieldbus usually presents functionalities according to some of the different layers of the Open System Interconnection model. In any case, physical layer and data link layer are always necessary for the fieldbus architecture. The standardization of protocols is far from being finished, and researchers from different countries around the world are devoting significant efforts to achieve better performances like delays, delay variability, and determinism in the context of networked real-time systems for distributed control applications [3], [4]. Recently, general data networks such as Ethernet, even using wireless links, are rapidly advancing to be used as control networks for applications at some higher levels in factory infrastructure [5], [6]. In general, very little consideration is devoted to the power supply transmission to distributed devices and to a system's sensitivity to noise.

Our main aim is to present solutions to these problems, not to propose a new fieldbus standard. In fact, our contributions can be applied to existing fieldbuses to improve their flexibility and robustness.

If we consider the five-level model of application in the hierarchical architecture for Computer Integrated Manufacturing defined by the National Bureau of Standards [7], we should specify that the main concern of this paper relates to the socalled machine control level. At this level, the fieldbus network 
is used to interconnect the sensors and actuators to the machine controllers, where a central controller is usually used for local supervisory control and even for interconnections with other fieldbuses that may exist at other higher levels like cell control level or factory control level.

Section II is devoted to the reduction of the costs of wiring. A traditional approach is to include power delivery to remote devices with the bus. Important aspects such as the amount of energy available or the cost of the cable are considered.

Section III describes how the power delivery capacity of a fieldbus can be reinforced by including a traditional supply of $24 \mathrm{~V}$ dc and also a novel secondary $24 \mathrm{~V}$ ac. This improves the technoeconomic advantages of powered fieldbuses facilitating direct powering of the distributed $\mathrm{I} / \mathrm{O}$ electronic modules and field devices, too. This avoids extra costs associated to the additional cabling frequently needed in conventional fieldbus systems.

As we will see, power distribution through the bus and conducted noise disturbances are strongly related. Section IV analyzes the electric perturbations usually appearing in industrial environments and the corresponding derived noise disturbances conducted on the fieldbus cable. In this paper, this issue is faced, and a novel solution is offered. To improve the network noise immunity, the addition of one dedicated ground wire, the so-called "protective earth wire," presented is put forward and analyzed in Section V. A second additional ground wire, called "industrial earth wire," designed for the power return of distributed field devices like sensors and industrial actuators is also presented. Practical quantitative results are shown.

Section VI presents the implementation of these innovations, the new MBus-I/O, and an industrial application using this fieldbus: digital control of desalination water plants with capacities from 10 to $400 \mathrm{~m}^{3} /$ day [8]. Particular results and benefits obtained with the MBus-I/O application are described. Finally, in Section VII, this paper concludes with a summary on the improvements obtained and points out the interest of adopting some of the related innovative aspects in other existent industrial fieldbuses at the machine control level.

\section{TOWARD The Minimization OF WiRing Costs}

Although the multiple advantages of process automation networks are well known and have been widely accepted in industry, at machine control level, perhaps the simplest but most important advantage is the reduced volume of wiring. Regarding this subject, the use of a powered fieldbus notably reinforces these benefits: Field wiring is greatly simplified if power is distributed within the fieldbus cable. It brings down costs and increases system flexibility by allowing easy insertion of new I/O modules and field devices. Nevertheless, as it will be discussed later, the current capacity of existing powered fieldbuses is often quite limited so that connecting a few nodes could require additional separate cabling to supply power. In this case, a great part of the advantage of a single fieldbus is lost.

This is not a new idea. The old 4-20-mA signaling scheme allowed up to $4 \mathrm{~mA}$ of current to supply power to the sensor/actuator. Soon, the need for advanced diagnostics, calibration and local control, will lead to the emergence of the so-called intelligent instrumentation: devices with digital capability that could be communicated with a digital signal, superimposed on the analog 4-20-mA signal [2]. Meanwhile, serial networks were developed, and the benefits of the reduced cabling cost and increased functionality of field devices allowed the jump to the fieldbus model. Nevertheless, as the focus moved to the communication problem, power delivery to remote devices was almost forgotten in standards. Fortunately, some fieldbuses (e.g., Solartron, S-Net, ISIbus, AS-i, Foundation fieldbus, and Profibus PA) were developed with both issues in mind.

Another reason to avoid, including power in the bus, is the susceptibility of such systems to conducted noise (see Section IV). As a consequence, only a few of them support simultaneous data communication and distribution of power, but are limited to small currents (some milliamperes) [9]. Frequently, some engineering brochure warnings, like ". . fieldbus powered devices should strive for minimum power consumption, without negatively impacting desired functionality," are found. In the simplest $\mathrm{I} / \mathrm{O}$ industrial fieldbus category, the AS-Interface standard [10] proposes a powered fieldbus that can supply higher currents of some amperes, but imposing a physical layer constituted by an expensive special two-wire cable (with a cost exceeding ten times that of a standard multitwisted-pair cable), with restrictions on fieldbus segment length to a maximum of $100 \mathrm{~m}$ and with limitations on messages $(14 \mathrm{~b}$ only) that exclude or make the use of distributed intelligent I/O modules difficult.

In industry automation, as well as in vehicle automation using Controller Area Network fieldbus [11], the previously described power routing problem is often avoided by installing additional separated cabling to independently power those field devices with specific power requirements, as well as the I/O modules. If so, a great part of the advantage in wiring overhead reduction obtained by using a single cable would be lost.

Another approach is to fully do without wires. Wireless communications to field devices further reduce the volume of wiring needed, although frequent power is still required. Note that battery-powered solutions could have some important practical limitations, e.g., available power, battery life, and temperature range. In addition, reliability of wireless networks may represent a critical aspect because of interferences and fading errors. Thus, wireless systems cannot be thought of as a replacement of wired networks but, rather, as dedicated wireless extensions for wired networks [12], [13].

In fact, at the factory control level, the main supply can be accessible within the industrial plant, but at the machine control level, extra wiring cost is normally required for powering distributed electronic I/O modules as well as sensors/actuators. The advantages of wireless extensions are restricted to certain special industrial areas, like in thermocouple monitoring and in mobile device control, rotating equipment automation, and robots [14], [15].

In spite of the different standards related to fieldbuses, most of them are based on a physical medium composed of two to five wires for digital data transmission, having a maximum data rate between some kilobits per second to some megabits per second, and having in certain cases an additional wire for 


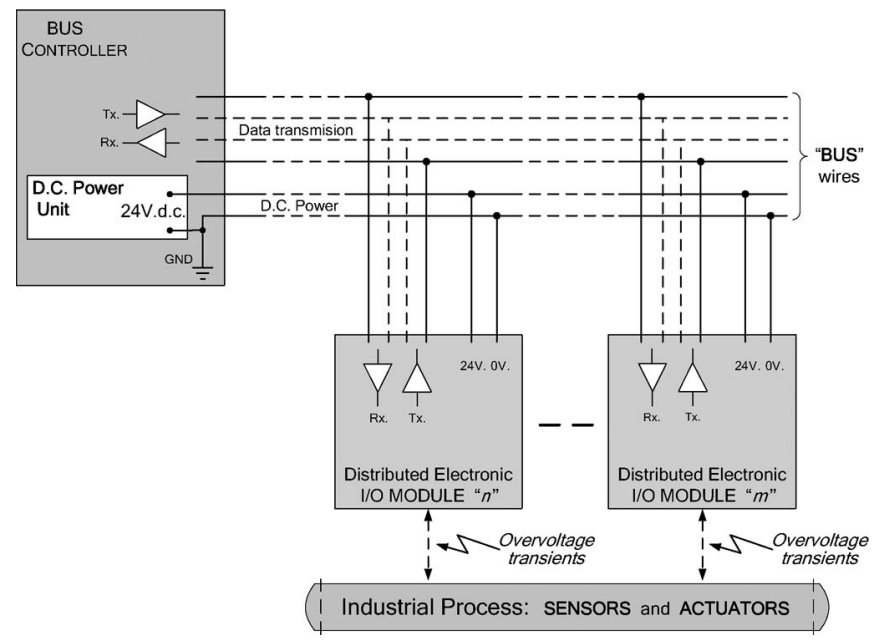

Fig. 1. Conventional networked control system.

TABLE I

Price of Popular Fieldbus Cables (SOURCE: FARNELl CATALOG 2010 EDITION)

\begin{tabular}{|l|l|r|}
\hline \multicolumn{1}{|c|}{ Fieldbus } & \multicolumn{1}{|c|}{ Wires and size } & Price $(\boldsymbol{€} / \mathbf{m})$ \\
\hline Ethernet & $4 \times 2 \times 24 \mathrm{AWG}$ & 0.70 \\
\hline Interbus & $2 \times 3 \times 18 \mathrm{AWG}$ (shielded) & 8.56 \\
\hline Profibus PA & $2 \times 22 \mathrm{AWG}$ (shielded) & 3.11 \\
\hline DeviceNet & $2 \times 2 \times 24$ AWG (shielded) & 6.00 \\
\hline AS- $\mathrm{i}$ & $2 \times 16 \mathrm{AWG}$ & 6.11 \\
\hline
\end{tabular}

carrying some limited power to the distributed equipment, typically at $24 \mathrm{~V} \mathrm{dc}$. Sometimes, a sole pair carries data and power. The physical medium choice and its powering capability have an important practical impact on the performance and cost of the complete fieldbus control system. Thus, this paper is focused on the physical medium design in view to enlarge the power fieldbus capacity for getting maximum flexibility and economy of the whole system and, at the same time, to improve the immunity of the distributed modules against conducted electrical disturbances. In fact, a conventional twisted-pair fieldbus carrying data and power, like that shown in Fig. 1, remains too sensible to the electrical perturbations originated by overcurrent transients circulating over the own bus ground wire.

It is a usually accepted concept that wiring cost is directly related to the number and size of wires within the bus cable. Nevertheless, another important factor that can be even more determining is the multivendor availability of the cable. Thus, the AS-i cable, with two wires that carry power and data, is about $6 € / \mathrm{m}$. Compare this with the four-twisted-pair Ethernet CAT5 cable $(0.70 € / \mathrm{m})$. Table I summarizes cable costs for popular fieldbuses. The same can be applied to connectors.

\section{Power on the Bus CABle}

Although a great effort has been made in recent years by standardization bodies to find ways to integrate the electrical power on the same wires that carry information [2], it is difficult to find fieldbuses that supply enough energy for distributed intelligence and communication electronics. Moreover, it is almost impossible to see any power specifications in present

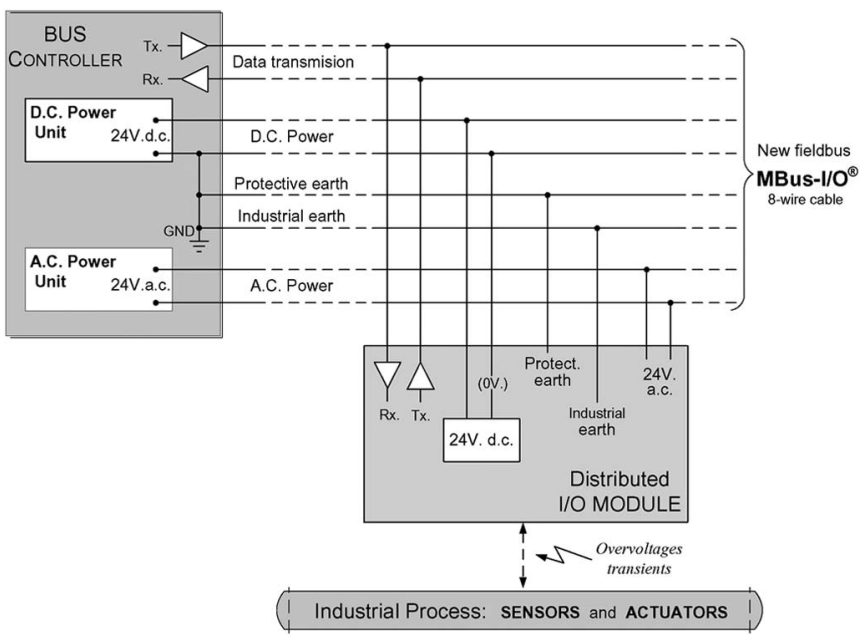

Fig. 2. New fieldbus medium composed of an eight-wire cable.

standards capable of fulfilling the widened power requirements needed to supply enough energy to the final application of distributed modules.

This paper explores a different method to improve a system's flexibility in order to satisfy these requirements. The bus brings $24 \mathrm{~V} \mathrm{dc}$ (mainly to power electronic I/O modules) and $24 \mathrm{~V} \mathrm{ac}$ (mainly to power sensors/actuators) to the remote modules by adding wires to the bus cable. The simplified electronics (no need of modulation schemes) and the extra power available for the distributed modules making up for the possible higher cost of a more complex wire are proven. Moreover, as have been seen, an eight-wire cable can be cheaper than a two-wire one.

As a result, the fieldbus physical medium proposed is a fourtwisted-pair cable as shown in Fig. 2 (one pair for balanced half-duplex data transmission, one for $24 \mathrm{~V} \mathrm{dc}$, and one for $24 \mathrm{~V} \mathrm{ac}$ ). In fact, this kind of cable, analogous to the fourtwisted-pair cable used for Ethernet communications, is fitted to an industrial standard and thus leading to a low-cost very competitive design.

Note that the $24-\mathrm{V}$ dc power is directly transmitted by a pair of wires and does not require any special modulation technique with power conditioners, as it is done by other fieldbuses, that contributes to limit the fieldbus power capacity and/or to increase the cost. Some other fieldbuses, like the wellknown MODBUS [16], optionally consider the possibility of adding one supplementary wire for power supply (5.24 V dc), although using the same single wire for "signal common and power common," so that the bus power capacity should remain strongly restricted. In this solution, this limitation will be overcome by taking advantage of the spare twisted pair of the cable (see Section V).

The addition of a secondary ac power supply constitutes another innovation of the paper mainly devoted to facilitate the use, by simple and inexpensive connection, of field elements that work easier with ac power. Some ac elements, such as electromagnets, electric motors, etc., are often more competitive than dc ones. Moreover, the bus ac power line facilitates design of galvanic isolated devices, as measurement converters, by using small and simple transformers and thus avoiding noisy switched techniques. 


\section{Electric Disturbances in Fieldbuses}

Currently, there exists much literature on industrial control networks, but seldom can one find any communication dealing with the fieldbus sensibility, as well as the protection techniques recommended, against the high-voltage/highfrequency interference pulses generated by the switching of inductive industrial loads and by lightning phenomena. This interfering conducted noise, originating typically in industrial plants by the on/off control of power appliances, disturbs every distributed module connected to the bus and originates trouble in the dispersed electronic microcontrollers that could adopt undefined or a dangerous operating state. The severity of these disturbances varies with the type of industry, and in some cases, the risk of troubles is so high that the control network has been built using an optical fiber communication link [17] in order to ensure enough noise immunity. Nevertheless, this approach should not be generalized because of the network cost increase due to the use of some expensive communication elements and because of the additional wiring required to power the devices.

These interferences occur anywhere electrical currents are switched by electromechanical devices: circuit breaker operation, pump start/stop, ..., and unbalanced ground transients among far away located devices are common causes of highvoltage disturbances. Typically, these electric disturbances affect the electronic control system by direct coupling through the main supply and by capacitive coupling to the input/output circuits (sensors and actuators) originating high-voltage/highfrequency interference pulses having voltage peaks over $100 \mathrm{~V}$, sometimes reaching $1000 \mathrm{~V}$ and more, and frequencies from kilohertz to some megahertz. In electromagnetic compatibility (EMC) regulations for industrial-process measurement and control equipment, these kinds of interferences are characterized by electrical fast transient burst as specified in the IEC 61000-4-4 Standard [18]. Note that, in this paper, radiated electromagnetic interferences that usually originate small disturbances of much less importance (in industrial control systems) are not considered. Protections against these disturbances on power supply ports may be defined rather easily, and there are well-known techniques for power line filter design [19], [20].

However, concerning I/O ports, the task becomes tougher. Thus, this communication is focused on the electric fast transient burst disturbances, usually common mode capacitively coupled, acting on the many input/output circuits distributed through the industrial plant. The authors have much experience in these kinds of disturbances, frequently appearing in most types of industry [21], [22], which become particularly severe in the case of networked control systems because the far away location of distributed electronic modules over the plant increases the risk of higher transient voltage differences. System disturbances are extremely sensible to routing design of the many interconnections and to the layout of wires.

Thus, every distributed electronic module must be designed to withstand those overvoltage transients to keep the equipment in good working order. This requirement is usually accomplished by adding some transient protection electronic components (like caps, Zener diodes, varistors, etc.) to the modules in order to provide a low-impedance path to ground for shunting the disturbance and to avoid equipment malfunction.

However, with powered fieldbuses as shown in Fig. 1, the distributed electronic modules (for control purposes or simply for I/O concentrating points) connected to the fieldbus suffer a decrease in their immunity to the previously mentioned I/O electric perturbations, since the disturbance path to ground is the own common circuit of the bus (used as $0 \mathrm{~V}$ for the power supply distributed by the fieldbus). Thus, each transient generates a conducted noise along the $0-\mathrm{V}$ fieldbus wire, thus transmitting interference effects to every electronic module powered by the same fieldbus branch.

Indeed, the long GND bus wire ("common"), with the associate plugs, is far from being a low-impedance circuit due to parasite resistances and inductances along the bus cable, and the extended practice of using twisted-pair cables contributes to increased equivalent inductances. Hence, the conducted noise is responsible for overvoltage transients that propagate along the bus GND wire. Since this wire is also the return path for the power supply of every distributed module, they all receive a noisy " $0-\mathrm{V}$ " power supply that can cause malfunctions or even block them. The network system's operation turns out for an appreciably decrease in reliability and security affecting the industrial plant. Typically, the system should need to reset the affected modules, and in certain cases, replacement of modules may be required if they were permanently damaged.

Apart from the previous conducted noise, additional electromagnetic inducted noise can appear on the fieldbus too, originated by induced influences from equipment in close proximity to the bus cable. The twisted pair is widely used in control networks to reduce magnetic noise pickup, although it reduces data transmission capability to some $100 \mathrm{kHz}$ maximum. Additionally, if twisted pairs are shielded, the protection against capacitive pickup should be improved, but the loss of flexibility and the increase of the weight and cost of the cable can be a serious disadvantage. Despite this, in industrial network applications, the interfering inducted noise level can be quite reduced by a careful planning of the bus cable layout along the industrial plant (i.e., avoiding parallel running to other power lines). In addition, at this machine control level, the volume of data to be transmitted is usually quite limited and low-speed communications (far less than $100 \mathrm{kHz}$ ) should be enough, so that low-pass filtering can be applied to obtain additional inducted noise reductions. Applying such good EMC design practices in industrial network applications, at machine control level, it can be assumed that the inducted noise usually shows much lower energy level than the previously considered conducted noise. Hence, this paper will concentrate on noise reduction techniques for the conducted noise in industry powered fieldbus cables.

\section{Dealing With Disturbances}

\section{A. Protective Earth}

With regard to the previously analyzed fieldbus conducted electric disturbances in networked control systems, the innovation furnished by this paper entails improving system's 


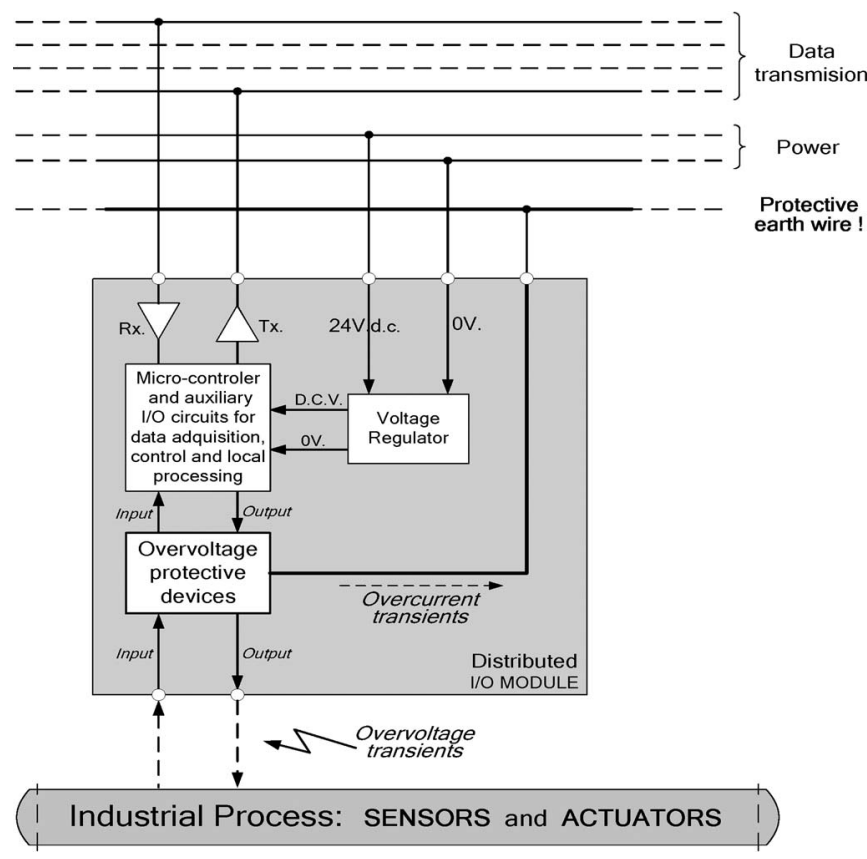

Fig. 3. Fieldbus medium with a new additional earth wire.

inmunity to this conducted noise by splitting the GND wire of the conventional fieldbus cable into two wires: power ground and "protective earth" (see Fig. 3). The former is associated to the $24-\mathrm{V}$ dc power line, establishing the " $0-\mathrm{V}$ " current return path for the distributed modules' power supply, and the latter is a separated return path, exclusively for evacuating the conducted noise, to be used by the protective circuits located at input/output modules' ports.

The contribution of the added protective earth wire consists in providing, for every distributed electronic module, an independent path to be used for shunting to earth those overvoltages usually appearing on the input/output connections to the industrial process. This removes overcurrents in the power ground wire $(0 \mathrm{~V})$ and avoids disturbing every electronic module connected to the fieldbus through its own "electronic $0-\mathrm{V}$ " power supply circuit. Note that this improvement should remove malfunctions and blockings often noticed on conventional distributed control equipment.

A simple numerical example as follows will help to understand problem's magnitude.

Typical overvoltage on I/O connections:

Amplitude: $1000 \mathrm{~V}$

Frequency: $1 \mathrm{MHz}$

Source eq. impedance: $200 \Omega$

Typical fieldbus wire electrical characteristics:

Bus length: $10 \mathrm{~m}$

Lineal resistance: $20 \mathrm{~m} \Omega / \mathrm{m}$

Connector resistance: $0.25 \Omega$

Lineal inductance: $500 \mathrm{nH} / \mathrm{m}$

Hence, at $1 \mathrm{MHz}$, ground wire's impedance is

$$
\begin{aligned}
/ \mathbf{Z} / & =\left[(10 \times 0.02+0.5)^{2}+\left(2 \pi 10^{6} \times 10 \times 0.510^{-6}\right)^{2}\right]^{1 / 2} \\
& \approx 32 \Omega
\end{aligned}
$$

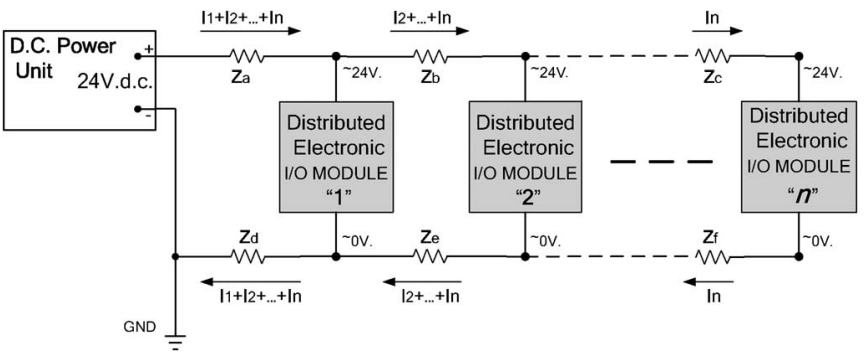

Fig. 4. Power bus with load interactions.

and we can deduce that the overcurrent transient flowing through the bus ground wire will be as high as

$$
I_{\text {peak }} \approx 1000 \mathrm{~V} /(200+32) \approx 4.3 \mathrm{~A}
$$

and thus, the $I Z$ drop along the mentioned fieldbus ground wire will be

$$
V_{\text {peak }} \approx 4.3 \mathrm{~A} \times 32 \Omega \approx 140 \mathrm{~V} .
$$

Thus, dangerous high-level voltage pulses are obtained. When appearing on the power line ground wire (" $0 \mathrm{~V}$ ") of the conventional fieldbus, these pulses can affect normal operation of the electronic distributed I/O modules.

However, with the proposed solution, the $140-\mathrm{V}$ pulses would propagate through the separate protective earth wire, not affecting the power line ground ("electronic $0 \mathrm{~V}$ ") wire and thus keeping the I/O modules in good working order.

\section{B. Industrial Earth}

Furthermore, another conducted noise may also appear on powered fieldbuses, quite similar to those considered previously, but now created by interaction of the normal operation loads at the multiple distributed I/O modules. The simplified circuit model in Fig. 4 shows the load currents that flow through the positive power bus and power return conductors exhibiting finite impedance.

The power bus $I Z$ drops create $\mathrm{I} / \mathrm{O}$ module interaction, acting as a conducted noise in the " $0-\mathrm{V}$ " power return wire, so that some instabilities or falsely triggered digital circuits can occur. In principle, these interactions can be minimized by using low-power electronics [23], but it becomes an unquestionable disturbance source if the same power bus also supplies current for operating relays, dc motors, and other I/O devices with nonnegligible loads. By an on/off control of such devices, high-current variations will appear that again should generate high-voltage transients along the distributed power line.

To overcome this problem, the following can be performed: 1) Minimize power drained by the system, and 2) derive the return path of heavy loads through a new path. As one of the goals is to maximize power available to remote elements, the inclusion of one additional earth wire, that is named "industrial earth," is proposed (see Fig. 2). Note that there was a spare pair in the cable, so the use of an additional earth wire constitutes a low-cost solution. Distributed modules should use this "industrial earth" as a separate return path for the power drained by the field devices powered by the own fieldbus. 


\section{Application to Water Desalination Plants}

\section{A. System Design}

The IEA Research Group (Electronic Instrumentation and Applications) of the University of Seville has been working since 1997, under the leadership of Professor Menéndez, on advanced automation of water desalination plants by reverse osmosis, in order to react to the "lack of an adequate response" detected by the commercial sector of industrial brackish water desalination plants. Indeed, the existing industrial desalination plants are usually manufactured in a "tailor-made" manner, with a minimum level of automation in order to limit manufacturing costs, but with poor adjustment capabilities and with high maintenance costs.

Therefore, the IEA Research Group developed some prototypes of desalination plants with integrated digital control, using fieldbus technology in order to minimize costs, but with a maximum of robustness. All the innovations described in the previous sections around a simple communication link were named MBus-I/O.

MBus-I/O data communication is half-duplex RS485 signaling with a character-based asynchronous transmission and master-slave medium access control. Data frames are 8-32 B long. Speed communication is $9.6 \mathrm{~kb} / \mathrm{s}$, enough for the time constraints and volume of data required. The physical medium used is a standard Ethernet CAT 5 cable, i.e., four-twisted-pair unshielded cable, with 24 AWG conductor size and low-cost standard RJ45 plugs. Note that, because of plugs and twistedpair restrictions, the maximum current capacity will be limited to $1.5 \mathrm{~A}$.

It is not the scope of this paper to describe in detail these desalination plants, but the intention is only to use this industrial application as a significant example to illustrate the benefits of the new MBus-I/O. Currently, a Spanish company is manufacturing industrial brackish water desalination plants using the digital control technology developed by the IEA Research Group [8], [24].

The control system is based on a set of distributed smart electronic I/O modules connected through MBus-I/O to a small PLC used as a central control unit. Every I/O module is equipped with a powerful microcontroller, specifically designed to put "intelligence" near sensors and actuators for built-in control facilities with anomaly detection [25], and the PLC has some wireless facilities for remote supervisory control, enabling effective maintenance at minimum cost. The system must withstand strong power disturbances frequently found in isolated rural places, with poor ac power lines and suffering major transient disturbances on the occasion of atmospheric discharges. The water conductivity contributes to spray the overvoltage transients over the distributed electronic I/O modules.

Fig. 5 shows a desalination plant, with a capacity of $28 \mathrm{~m}^{3} /$ day of permeated water, housed in a metal cabinet (about $80 \times$ $60 \times 200 \mathrm{~cm}^{3}$ ) containing the reverse osmosis membranes, filters, pumps, and valves with pipes and water tanks.

The control system has to measure at least three flows, three pressures, and one conductivity, with the detection of many water levels, and has to control a minimum of two pumps,

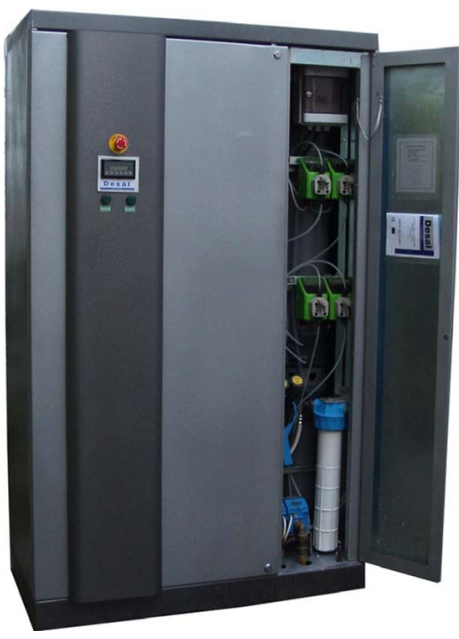

Fig. 5. Desalination plant with MBus-I/O distributed digital control.

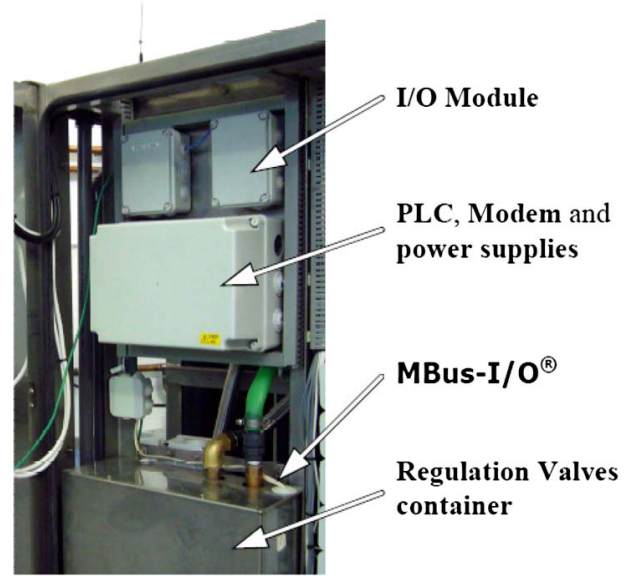

Fig. 6. Inside the desalination plant: View on some electronic modules and cabling details of the MBus-I/O.

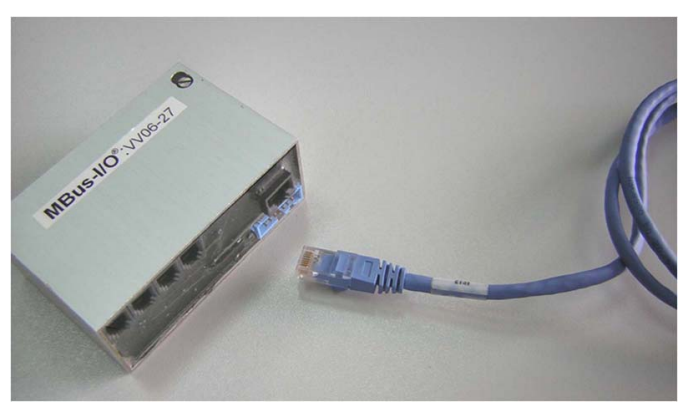

Fig. 7. MBus-I/O distributed electronic module for smart regulation of simple low-cost valves.

three regulation valves, three on/off valves, and some dosage devices, all of them scattered throughout the interior of the cabinet.

In Fig. 6, one can appreciate inside the cabinet some different components and some wiring details. In Fig. 7, the MBus-I/O cable is shown together with one of the electronic IO modules [26], in an IP-53 enclosure, as an example of these used in the fieldbus network control of the desalination plant. Industrial 


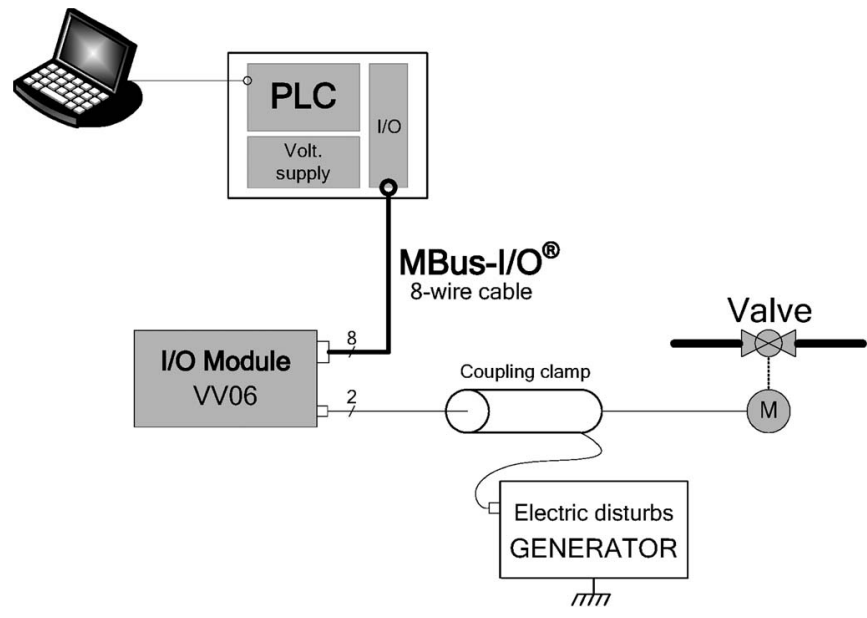

Fig. 8. Testing MBus-I/O system with fast transients.

grade protections IP55-IP56 are attained by adding low-cost small plastic enclosures.

The availability of a $24-\mathrm{V}$ ac power on the same MBus-I/O cable has facilitated the use of low-cost electrovalves, cheaper than the dc powered equivalents. It has also allowed a simple and noise-free design, using single small transformers, for the implementation of the galvanic isolation required at the I/O modules for measurement input connections. Note that the galvanic isolation is absolutely vital for those field devices installed in industrial plants dealing with water like desalination plants, because it provides a conductive medium for transmitting disturbances and unbalanced ground perturbations.

The system design has taken special care to reduce electrical consumptions [23]. Thus, the ensemble of all electronic I/O modules with sensors and valves operates with a maximum power consumption below $30 \mathrm{~W}$ dc and $30 \mathrm{~W}$ ac. Therefore, every element is simply powered from the bus, avoiding any extra wiring costs, except for those elements, like pumps, that require larger quantity of power at $230 \mathrm{~V}$ ac.

\section{B. Practical Results}

The use of MBus-I/O, incorporating "data+power fieldbus distribution," has led to an extreme wiring simplicity, including error-free "Plug and Work" facilities for component installation and replacement. Without extra cost, the MBus-I/O has also been a key factor for the improvement of $\mathrm{I} / \mathrm{O}$ overvoltage transient immunity, even though power is transmitted through the own bus cable.

The excellent electrical system protection achieved has been tested with fast transient voltages, according to the IEC610004-4 Burst Standard, applying common-mode disturb voltages through a capacitively coupling clamp [27] onto the I/O wires, in close proximity to the distributed I/O modules interconnected by the bus.

The diagram shown in Fig. 8 illustrates, as an example, the test configuration used to apply high frequency/high voltages to the electronic module VV06 [26] dedicated for valve control. According to the EMC regulation IEC61000-4-4 [18], the

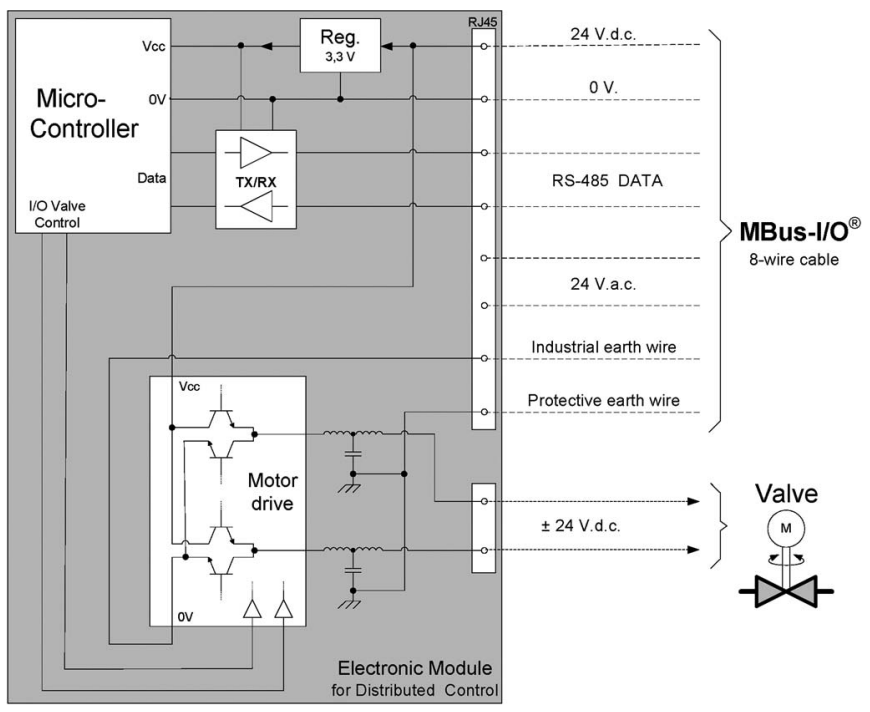

Fig. 9. I/O Module VV06 for "intelligent" valve control and flow regulation with MBus-I/O connections.

TABLE II

IEC 61000-4-4 TEST ON I/O MODULE VV06

\begin{tabular}{|l|l|l|}
\hline Voltage peaks & \multicolumn{1}{|c|}{ Configuration $\mathbf{A}$} & \multicolumn{1}{|c|}{ Configuration B } \\
\hline $800 \mathrm{~V}$ & No incidents & No incidents \\
\hline $900 \mathrm{~V}$ & No incidents & Some resets appear \\
\hline $1000 \mathrm{~V}$ & No incidents & Frequent resets \\
\hline $1100 \mathrm{~V}$ & No incidents & Always resets \\
\hline $1200 \mathrm{~V}$ & Some resets appear & Always resets \\
\hline
\end{tabular}

interference applied is burst pulses with $500-1000-\mathrm{V}$ pulse amplitude, with 5/50-ns pulse form and with 5-kHz pulse repeating frequency.

Fig. 9 shows the electrical simplified scheme of the module VV06 under test with fieldbus and I/O connections. This scheme clearly manifests the different roles of the three earth wires supplied by the MBus-I/O ("electronic 0 V," "protective earth," and "industrial earth") and their relation to the control wires of the valve submitted to the disturbances.

Test results with high-frequency burst disturbances showed very satisfactory operations, even with $1000-\mathrm{V}$ voltage peaks: During the perturbation, no abnormal behavior on the I/O module was noticed, and after the perturbation, the electronic module continued to operate correctly (configuration A). However, the same experiments, repeated after the removal of the wire protection of the bus (shorting the "protective earth" wire to the "electronic $0 \mathrm{~V}$," corresponding to configuration B), showed a significantly degraded performance. Experimental results are included in Table II.

In practice, these MBus-I/O robustness improvements were the key to achieving nonstop operation of the desalination plant without any trouble in harsh environments, even under lightning perturbations.

\section{CONCLUSION}

In this paper, a new physical layer for industrial fieldbus networks at machine control level has been presented, the 
MBus-I/O, consisting of an eight-wire cable with one twisted pair for data transmission, two pairs for power delivery, and one pair for protective earth wires, as is shown in Fig. 2. Although MBus-I/O incorporates some extra wires, its cost remains significantly lower than other fieldbuses because it simply uses a standard low-cost unshielded four-pair cable with RJ45 plugs (same bargain standard cable used for Ethernet applications).

An advantage of this MBus-I/O is to provide a powered fieldbus that distributes, on the same low-cost fieldbus cable, two $24-\mathrm{V}$ power lines, dc and ac, to improve flexibility and to allow easy inclusion of ac powered elements, sometimes more efficient than dc powered ones. Separated cables for power are not required, so that an additional important reduction of the wiring is obtained, in order to lower the overall control system costs, particularly in these industries where it is hard to find power supply spots anywhere in the facilities.

Although using a simple unshielded cable, the other contribution of the MBus-I/O relies on the high system immunity obtained against the conducted noise, usually appearing in almost every industry fieldbus, originated by fast transient disturbances at the $\mathrm{I} / \mathrm{O}$ ports.

The fact is that, because of the $I Z$ drops, conducted noise is even more harmful in systems using powered fieldbuses, so that, in practice, only very limited industrial applications distribute power within the fieldbus. In the powered MBus$\mathbf{I} / \mathbf{O}$, it is important to emphasize that it significantly improves the system robustness by simply adding two new low-cost earth wires in the own fieldbus cable.

This new fieldbus has been successfully employed in the manufacturing of automated desalination water plants, allowing integration of the whole plant and its control components, with the corresponding wiring, in a small cabinet. A remarkable cabling reduction of over $85 \%$ has been obtained, which can be compared to the $60 \%$ reduction of cables typically obtained by coachbuilders using the CAN nonpowered fieldbus [11].

At present, distributed automation systems are widely used, and the proposed MBus-I/O does not claim to replace any existent industrial fieldbus. Nevertheless, the work presented here describes a convenient method, easy to apply in practice, for different types of industries, to enlarge fieldbus power capacity and to improve distributed system noise immunity by a simple low-cost fieldbus physical layer adaptation.

Furthermore, the experimental results showed that the IEC 61000-4-4 (level 3) standard, applied to I/O ports, is adequate to test real disturbance immunity in industrial distributed electronic I/O modules. As a result, this electrical fast transient/burst EMC test should be strongly recommended for the verification of robustness in industrial control systems, particularly for those using powered fieldbuses.

\section{REFERENCES}

[1] J. P. Thomesse, "Fieldbus technology in industrial automation," Proc. IEEE, vol. 93, no. 6, pp. 1073-1101, Jun. 2005.

[2] J. R. Jordan, Serial Networked Field Instrumentation. New York: Wiley, 1995.
[3] M.-Y. Chow, "Special section on distributed network-based control system and applications," IEEE Trans. Ind. Electron., vol. 51, no. 6, pp. 1126-1279, Dec. 2004.

[4] P. Antsaklis and J. Baillieul, "Special issue on networked control systems," IEEE Trans. Autom. Control, vol. 49, no. 9, pp. 1421-1423, Sep. 2004.

[5] J. R. Moyne and D. M. Tilbury, "The emergence of industrial control networks for manufacturing control, diagnostics, and safety data," Proc. IEEE, vol. 95, no. 1, pp. 29-47, Jan. 2007.

[6] H. Kleines, S. Detert, M. Drochner, and F. Suxdorf, "Performance aspects of PROFINET IO," IEEE Trans. Nucl. Sci., vol. 55, no. 1, pp. 290-294, Feb. 2008.

[7] P. F. Brown and C. R. Mac Lean, "The architecture of the NBS factory automation," in Proc. IFAC Congr., Münich, Germany, 1987.

[8] A. Menéndez, "Innovación tecnológica en plantas desaladoras de ósmosis inversa," Técnica Industrial, vol. 3/06, pp. 47-50, Jun. 2006.

[9] Standard: Industrial Communications Networks-Fieldbus Specifications-Part 2: Physical Layer Specification and Service Definition, IEC 61158-2, 2007.

[10] "AS-interface standard," Actuator and Sensor Interface, 2008. [Online]. Available: http//www.as-interface.net

[11] P. Marino, F. Poza, M. A. Dominguez, and S. Otero, "Electronics in automotive engineering: A top-down approach for implementing industrial fieldbus technologies in city buses and coaches," IEEE Trans. Ind. Electron., vol. 56, no. 2, pp. 589-600, Feb. 2009

[12] G. Cena, A. Valenzano, and S. Vitturi, "Hybrid wired/wireless networks for real-time communications," IEEE Ind. Electron. Mag., vol. 2, no. 1, pp. 8-20, Mar. 2008.

[13] L. Seno, S. Vitturi, and C. Zunino, "Analysis of ethernet powerlink wireless extensions based on the IEEE 802.11 WLAN," IEEE Trans. Ind. Inform., vol. 5, no. 2, pp. 86-98, May 2009.

[14] A. Flammini, D. Marioli, E. Sisinni, and A. Taroni, "Design and implementation of a wireless fieldbus for plastic machineries," IEEE Trans. Ind. Electron., vol. 56, no. 3, pp. 747-755, Mar. 2009.

[15] J. Kjellsson, A. E. Vallestad, R. Steigmann, and D. Dzung, "Integration of a wireless I/O interface for PROFIBUS and PROFINET for factory automation," IEEE Trans. Ind. Electron., vol. 56, no. 10, pp. 4279-4287, Oct. 2009.

[16] MODBUS Serial Line Specification and Implementation Guide V1.02002. [Online]. Available: http//www.modbus.org

[17] J. Garcia, F. R. Palomo, A. Luque, C. Aracil, J. M. Quero, D. Carrion, F. Gamiz, P. Revilla, J. Perez-Tinao, M. Moreno, P. Robles, and L. G. Franquelo, "Reconfigurable distributed network control system for industrial plant automation," IEEE Trans. Ind. Electron., vol. 51, no. 6, pp. 1168-1180, Dec. 2004.

[18] Standard: Electromagnetic Compatibility for Industrial-Process Measurement and Control Equipment-Part 4-4: Electrical Fast Transient/Burst Immunity Test, IEC 61000-4-4, 2004

[19] W. H. Lewis, "Recommended power and signal grounding for control and computer rooms," IEEE Trans. Ind. Appl., vol. IA-21, no. 6, pp. 15031516, Nov. 1985

[20] M. Kumar and V. Agarwal, "Power line filter design for conducted electromagnetic interference using time-domain measurements," IEEE Trans. Electromagn. Compat., vol. 48, no. 1, pp. 178-186, Feb. 2006.

[21] A. Menéndez, "Control of electricity distribution: The use of a minicomputer for each substation," in Proc. CIRED, Liege, Belgium, May $14-18,1979$.

[22] A. Menéndez, "Small capacity remote control system with multimicroprocessor structure," in Proc. I.F.A.C. Symp. Compon., Instrum. Tech. Low Cost Autom. Appl., Valencia, Spain, Nov. 1986.

[23] A. Barbancho, M. Castaño, A. Menéndez, and F. Simón, "Diseño de un bus de campo con requisitos de mínimo consumo," Rev. Automática e Instrumentación, vol. 32, pp. 77-84, Oct. 2001.

[24] A. Menéndez, D. Martín, D. F. Larios, and E. Personal, "Low-cost portable industrial supervision for small water treatment plants," in Proc. Int. Congr. Wastewater Small Communities SMALLWAT II, Seville, Spain, Nov. 11-15, 2007, pp. 187-188.

[25] A. Menéndez, F. Biscarri, A. B. Sanchez, and A. A. Gomez, "Uncertainty and redundancy in flow metrology," in Proc. II Colloque A\&E, SaintEtienne, France, Jul. 4-6, 2001.

[26] A. Menéndez, M. A. Leal, G. Domínguez-Adame, and E. Yaglián, "Motorización de válvulas de bajo coste mediante micro-controlador," Rev. Tecnología del Agua, vol. 250, pp. 50-54, Jul. 2004.

[27] F. Musolino and F. Fiori, "Modeling the IEC 61000-4-4 EFT injection clamp," IEEE Trans. Electromagn. Compat., vol. 50, no. 4, pp. 869-875, Nov. 2008. 